\title{
Comparison of double disk synergy test, VITEK 2 and Check- MDR CT102 for detection of ESBL producing isolates
}

\author{
Fatima Numanovic ${ }^{1}$, Mirsada Hukic ${ }^{2}$, Zineta Delibegovic ${ }^{1}$, Nijaz Tihic ${ }^{1}$, Selma Pasic ${ }^{1}$, \\ Merima Gegic ${ }^{1}$
}

${ }^{1}$ Polyclinic for Laboratory diagnostics Department for microbiology University clinical centre Tuzla Tuzla, Bosnia and Herzegovina ${ }^{2}$ Department of Medical Sciences of the Academy of Sciences and Arts of Bosnia and Herzegovina, Sarajevo Bosnia and Herzegovina

Corresponding author: Fatima Numanovic

Mehmedalije Maka Dizdara B7/V/32 75000 Tuzla

Bosnia and Herzegovina tima333@hotmail.com

Tel.: + 38735303564

Fax.: + 38735250474

Received: 17 January 2013

Accepted: 7 May 2013

Copyright $(\odot 2013$ by

Academy of Sciences and Arts of Bosnia and Herzegovina.

E-mail for permission to publish:

amabih@anubih.ba
Objective. This study is to define the statistical significance for detection of ESBL producers by the double disk synergy test and molecular test (Check-MDR $\mathrm{CT}_{102}$ ), microdilution test (VITEK 2 with AES) and double disk synergy test (DDST), as well as the microdilution test and molecular test. Materials and methods. Phenotypic testing of 55 isolates Enterobacteriaceae (Escherichia coli (14/55), Klebsiella pneumoniae (34/55), Klebsiella oxytoca (3/55) and Proteus mirabilis (4/55) was performed by VITEK 2 Compact/AES. When this test showed positive results for the ESBL phenotype, then DDST with amoxicillin/clavulanate, ceftazidime, cefpodoxime, aztreonam, ceftriaxone and cefoxitin disks was performed along with Check-MDR $\mathrm{CT}_{102}$ which identified CTX-M, TEM and SHV $\beta$-lactamases. Results. Applying the $\mathrm{McNe}$ mar test, we determined that there was a statistically significant difference in the results of detection of ESBLs bacteria using DDST compared to molecular methods ( $95 \% \mathrm{CI}=41.92$ to $54.55 ; \mathrm{p}<0.0001$ ), as well as a DDST and VITEK 2/AES ( $95 \% \mathrm{CI}=40.13$ to 52.73; $\mathrm{p}<0.0001$ ). We did not find any statistically significant difference in the results of detection of ESBL producers using molecular techniques and VITEK 2/AES (CI=-4,43 to 5,36; $\mathrm{p}=1$ ). Also we did not find any statistical.. difference between the resistance to cefpodoxime and ceftriaxone (50/50) compared to the results of molecular tests. Conclusion. In routine daily testing, good detection of ESBLs bacteria, especially CTX-M can be obtained with phenotypic methods with VITEK 2/AES and by DDST with cefpodoxime, and ceftriaksone disks.

Key words: ESBL, DDST, VITEK 2/AES, Check-MDR CT ${ }_{102}$.

\section{Introduction}

Extended spectrum $\beta$ lactamases (ESBL) are a group of enzymes which have the capability of hydrolyzing third-generation cephalosporins and aztreonam (but not cephamycins and carbapenems) and which are sensitive to inhibitors such as clavulanic acid, sulbactam and tazobactam. They developed from point mutation of genes which code production of primordial TEM-1, TEM-2, or SHV-1 $\beta$ lactamases with replacement of the configuration of amino acids at an active site for these enzymes (1). In 1989, Philippon, Labia and Jacoby presented the first example where resistance to $\beta$-lactam antibiotics mediated by $\beta$ lactamases was a result of important changes in the spectrum of 
substrates of these enzymes (2). Due to their activity, they can hydrolyze a wide range of ß-lactam antibiotics, such as penicillins and cephalosporins. Besides Klebsiella pneumoniae and Escherichia coli, which represent the most important pathogens producing ESBLs, other ESBL producers, such as Klebsiella oxytoca, Enterobacter cloacae, Enterobacter aerogenes, Serratia marcescens, Citrobacter diversus, Providencia stuartii, Proteus mirabilis, Salmonella typhimurium, Pseudomonas aeruginosa, Burkholderia cepacia and Acinetobacter spp. have been registered lately.

To date, 890 various ESBLs have been discovered worldwide. They are classified according to two basic schemes: the molecular classification scheme according to Ambler and the functional classification system according to Bush-Jacoby-Medieros $(3,4)$.

Phenotypic testing of bacteria (disk diffusion and microdilution methods) is performed according to the recommendations of the Clinical and Laboratory Standards Institute in the Performance Standards for Antimicrobial Susceptibility Testing (M100S21 Vol. 31 No 1) which we used in our study, or the recommendations of the European Committee on Antimicrobial Susceptibility Testing (EUCAST), January 5, 2011, version $1.3(5,6)$. Using the disk diffusion method, a typical phenotypic profile of ESBLs shows resistance to third-generation cephalosporins (ceftazidime, cefpodoxime, ceftriaxone) and monobactams (aztreonam), an increase of the zone of inhibition for amoxicillin/clavulonate towards third-generation cephalosporins, and sensitivity to cefoxitin. Besides standard phenotypic methods, automated systems can also be used for the detection of resistance. VITEK 2 cards contain an ESBL test which monitors susceptibility of Escherichia coli and Klebsiella species to cefepime, ceftazidime and cefotaxime alone and in combination with clavulanic acid. The logarithmic reduction of growth within wells containing clavulanic acid and those which do not contain clavulanic acid indicates expression of an ESBL. The ESBL test, in combination with the VITEK 2 Advanced Expert System (AES), represents a very sensitive methodology for detection of ESBLs in clinical isolates. AES indicates possible inconsistent results in the antibiogram and, using existing knowledge about resistence mechanisms and CLSI standards, it indicates a phenotype for each isolate. Examples of phenotypes detected by AES are: ESBL, AmpC, carbapenemases (metallo or KPC $\beta$ lactamases), as well as co-existing mechanisms of resistence (ESBL and AmpC) in the same isolate, where it may be necessary to apply molecular detection methods. The majority of VITEK 2 results are available on the same day that the VITEK 2 card is set up and VITEK 2 gives comments about the susceptibility test results, which can be used as guidelines by a clinician for antibiotic therapy.

Molecular diagnosis of ESBL is performed by the application of various methods, such as PFGE (pulsed field gel electrophoresis), multiplex PCR (polymerase chain reaction), sequencing of deoxyribonucleic acid (DNA) or pyrosequencing. One of the most sensitive methods, which is based on multiplex PCR and provides results in 24 hours, is the Check Points method. The principle of the Check Points diagnostic system is based on molecular recognition of the amplified target DNA sequence and subsequences with universal primers. The test uses highly-specific DNA markers necessary for differentiation of "real" ESBLs from non-ESBL variants TEM and SHV. This test detects and differentiates genes of different CTX-M families of enzymes, as well as SHV and TEM. Unlike conventional phenotypic methods, this test enables results to be obtained in one day.

Since results of genotypic testing methods are a reliable indicator of the existence 
of genes for resistance due to the presence of ESBLs, this study aims to define statistical significance for detection of ESBL producers by the DDST and molecular test (CheckMDR $\mathrm{CT}_{102}$ ), microdilution test (VITEK 2 with AES) and DDST, as well as microdilution test and molecular test.

\section{Material and methods}

This research was conducted at the Polyclinic for Laboratory Diagnostics, Institute of Microbiology University Clinical Centre, Tuzla in Bosnia and Herzegovina. The research included 55 isolates of Enterobacteriaceae (Escherichia coli (14/55), Klebsiella pneumoniae (34/55), Klebsiella oxytoca (3/55) and Proteus mirabilis (4/55)) where production of extended spectrum betalactamases had been detected using the microdilution method in VITEK 2/AES [ASTGN27 card, (bioMérieux, Marcy l'Étoile, France)], according to the procedure contained in the manufacturer's instructions. In this study we used the Klebsiella pneumoniae ATCC 700603 strain as a positive control, and Escherichia coli ATCC 25922 strain as a negative control.

We examined each confirmed isolate with the double disk synergy test and molecular test. We applied the double disk synergy test $\left(\mathrm{BD} \mathrm{BBL}^{\mathrm{TM}}\right.$ Sensi-Disc ${ }^{\mathrm{TM}}$ Antimicrobial susceptibility Test Discs; Mueller Hinton agar, Liofilchem s.r.l. Bacteriology products) with disks cefpodoxime, ceftazidime, cefotaxime, ceftriaxone, aztreonam and cefoxitin according to recommendations by Thomson et al. (7) respectively, and we used $20 \mathrm{~mm}$ disk spacing.

Klebsiella pneumoniae, Klebsiella oxytoca and Escherichia coli were considered resistant to cefpodoxime disks of $10 \mu \mathrm{g}$ if the zone of inhibition was $\leq 17 \mathrm{~mm}$, and $\leq 22$ $\mathrm{mm}$ for Proteus mirabilis. Strains were considered resistant to: ceftazidime disks of 30 $\mu \mathrm{g}$ if the zone of inhibition was $\leq 22 \mathrm{~mm}$, ce- fotaxime disks of $30 \mu \mathrm{g}$ if the zone of inhibition was $\leq 27 \mathrm{~mm}$, ceftriaxone disks of $30 \mu \mathrm{g}$ if the zone of inhibition was $\leq 25 \mathrm{~mm}$, aztreonam disks of $30 \mu \mathrm{g}$ if the zone of inhibition was $\leq 27 \mathrm{~mm}$ (5). We considered the screening test as positive if the zones of inhibition for the mentioned antibiotics were resistant, except for the zone of inhibition regarding cefoxitin, which was in the susceptible zone, i.e. $\geq 18 \mathrm{~mm}$.

Genotyping of phenotypically confirmed ESBL isolates was performed with the multiplex PCR application of the commercially available Check-MDR CT102 (Check-Points Health BV) for detection of TEM, SHV and CTX-M genes according to the manufacturer's instructions. Before performing PCR, a DNA extraction from a pure culture of each isolate was made using the mini-kit QIAamp DNA (Qiagen, Sample \& Assay Technologies).

\section{Statistical analysis}

The commercial statistical program Arcus Quickstat Biomedical was applied for data entry and basic statistical analysis. We used the chi-square test (McNemar) and statistical significance was tested at the 0.05 level.

\section{Results}

By using the VITEK 2 Compact system with AES, 14/55 Escherichia coli, 34/55 Klebsiella pneumonie, 3/55 Klebsiella oxytoca and 4/55 Proteus mirabilis were identified in a total of 55 Enterobacteriaceae isolates. The ESBL phenotype was identified in 49 isolates (89.09\%) while ESBL/AmpC resistance phenotype was identified in 6 isolates (10.90\%) (Figure 1).

Double-disk synergy test and molecular method Check-MDR CT ${ }_{102}$ were performed for all isolates. A positive result was found in 50 isolates $(90.90 \%)$ tested by Check-MDR $\mathrm{CT}_{102}$ and in 21 isolates (38.18\%) tested by double-disk synergy test 


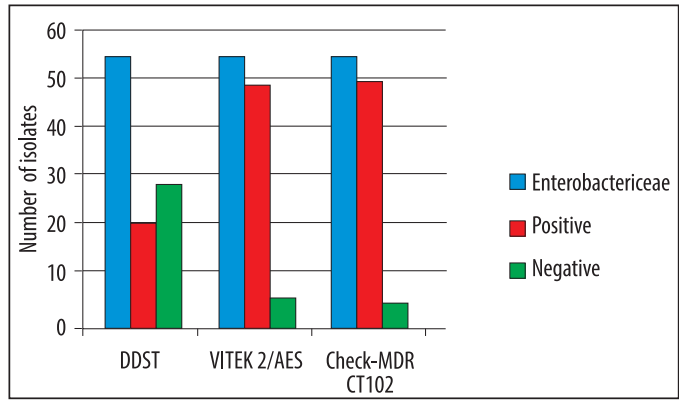

Figure 1 Results of ESBL detection by molecular, microdilution and DDST.

\section{Results obtained by the VITEK 2/AES}

Consecutive samples of positive ESBL isolates were taken, using VITEK 2/AES from our routine work. Out of 55 isolates of Enterobacteriaceae which were tested, 49 isolates tested by the VITEK 2 Compact system/AES were positive for phenotype ESBL. Out of those, $48 / 49$ also tested positive for phenotype by Check-MDR $\mathrm{CT}_{102}$. One isolate which was positive on this test did not prove positive for genotype of ESBL. Out of 6 isolates tested by the VITEK 2 Compact/AES and positive for a combined phenotype ESBL/AmpC, 2 tested by Check-MDR CT ${ }_{102}$ confirmed the presence of ESBL genotype (Figure 2).

\section{Results obtained by Check-MDR $C T_{102}$}

50 out of 55 isolates tested by Check-MDR $\mathrm{CT}_{102}$ were positive. In 49 isolates the group CTX-M1 beta-lactamases was identified and the group CTX-M9 was identified in the remaining one (Figure 3 ).

\section{Results obtained by the double-disk synergy test}

All 55 isolates were tested by the double-disk synergy test. A typical phenotype profile of ESBL was found in 21 (38.18\%). By considering the results of the molecular test CheckMDR CT $_{102}$ positive, we found a phenotypic profile in 21/50 (42\%) isolates. Furthermore,

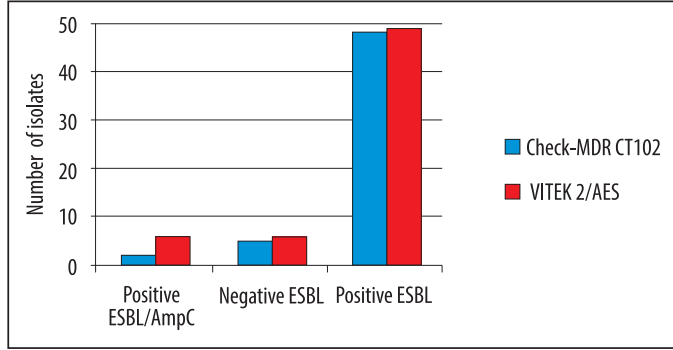

Figure 2 Results of the identification of ESBL using microdilution and molecular methods.

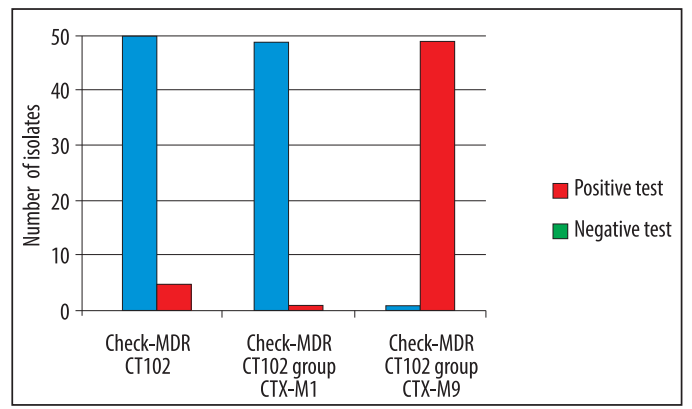

Figure 3 Illustration of ESBL genotypes.

we found 15 different phenotype profiles in the remaining isolates, 29/50 (58\%) (Table 1).

Out of 6 isolates which were positive (ESBL/ApmC) by the VITEK 2/AES, the phenotypic profile of AmpC beta-lactamases using DDST (when the diameter zone for ceftazidime, cefpotaxime, ceftriaxone or aztreonam was inside the zone of resistance, when there is no synergizing with clavulonic acid and when the organism is resistant to cefoxitin was found in 3 isolates) (Table 2).

\section{Comparison of results obtained by double disk synergy test, Check-MDR CT102 and VITEK 2/AES}

50 isolates tested by the molecular test were positive for genotype ESBL. Out of those, 21 were also positive by the double-disk synergy test. We found that the results of these two tests in detection of ESBL are significantly different $(\mathrm{CI}=41.92$ to $54.55 ; \mathrm{p}<0.0001)$. The sensitivity of double-disk synergy test was $48 \%$. 
Table 1 Phenotypic profile of ESBL isolates confirmed with Check-MDR $\mathrm{CT}_{102}$ and DDST negative

\begin{tabular}{|c|c|c|c|c|c|c|}
\hline \multirow{2}{*}{ Phenotypic profile ESBL } & \multicolumn{6}{|c|}{ Antibiotics } \\
\hline & AMC & CAZ & CPD & ATM & CRO & FOX \\
\hline I combination & $5 / \mathrm{S}$ & $5 / R$ & $5 / R$ & $5 / R$ & $5 / R$ & $5 / R$ \\
\hline Il combination & $9 / \mathrm{R}$ & $9 / R$ & $9 / \mathrm{R}$ & $9 / \mathrm{R}$ & $9 / \mathrm{R}$ & $9 / 1$ \\
\hline III combination & $1 / l$ & $1 / \mathrm{R}$ & $1 / R$ & $1 / 1$ & $1 / \mathrm{R}$ & $1 / \mathrm{R}$ \\
\hline IV combination & $2 / 1$ & $2 / 1$ & $2 / R$ & $2 / 1$ & $2 / R$ & $2 / R$ \\
\hline V combination & $1 / l$ & $1 / R$ & $1 / R$ & $1 / \mathrm{l}$ & $1 / \mathrm{R}$ & $1 / \mathrm{S}$ \\
\hline VI combination & $2 / \mathrm{R}$ & $2 / 1$ & $2 / \mathrm{R}$ & $2 / R$ & $2 / R$ & $2 / \mathrm{S}$ \\
\hline VII combination & $1 / I$ & $1 / \mathrm{S}$ & $1 / \mathrm{R}$ & $1 / \mathrm{R}$ & $1 / \mathrm{R}$ & $1 / \mathrm{S}$ \\
\hline VIII combination & $1 / l$ & $1 / \mathrm{S}$ & $1 / \mathrm{R}$ & $1 / \mathrm{S}$ & $1 / \mathrm{R}$ & $1 / \mathrm{S}$ \\
\hline IX combination & $1 / \mathrm{S}$ & $1 / l$ & $1 / \mathrm{R}$ & $1 / \mathrm{R}$ & $1 / \mathrm{R}$ & $1 / l$ \\
\hline X combination & $1 / I$ & $1 / /$ & $1 / \mathrm{R}$ & $1 / \mathrm{R}$ & $1 / R$ & $1 / l$ \\
\hline $\mathrm{XI}$ combination & $1 / /$ & $1 / \mathrm{S}$ & $1 / \mathrm{R}$ & $1 / \mathrm{S}$ & $1 / R$ & $1 / \mathrm{I}$ \\
\hline XII combination & $1 / l$ & $1 / I$ & $1 / R$ & $1 / l$ & $1 / R$ & $1 / \mathrm{S}$ \\
\hline XIII combination & $1 / \mathrm{R}$ & $1 / \mathrm{S}$ & $1 / \mathrm{R}$ & $1 / 1$ & $1 / R$ & $1 / \mathrm{S}$ \\
\hline XIV combination & $1 / R$ & $1 / 1$ & $1 / R$ & $1 / R$ & $1 / R$ & $1 / 1$ \\
\hline $\mathrm{XV}$ combination & $1 / R$ & $1 / R$ & $1 / R$ & $1 / 1$ & $1 / \mathrm{R}$ & $1 / 1$ \\
\hline
\end{tabular}

$\mathrm{AMC}=$ amoxicillin/clavulanate; $\mathrm{CAZ}=$ ceftazidime; $\mathrm{CPD}=$ cefpodoxime; $\mathrm{ATM}=$ aztreonam; $C R O=$ ceftriaxone; $F O X=$ cefoxitin.

Table 2 Phenotypic profile of ESBL/AmpC isolates detected by the VITEK 2, ESBL test in conjunction with AES

\begin{tabular}{lllllll}
\hline \multirow{2}{*}{ Phenotype } & Antibiotics & & & & \\
\cline { 2 - 7 } & AMC & CAZ & CPD & ATM & CRO & FOX \\
\hline I combination & $2 / \mathrm{R}$ & $2 / \mathrm{S}$ & $2 / l$ & $2 / \mathrm{S}$ & $2 / \mathrm{I}$ & $2 / \mathrm{S}$ \\
II combination & $1 / \mathrm{R}$ & $1 / \mathrm{l}$ & $1 / \mathrm{l}$ & $1 / \mathrm{S}$ & $1 / \mathrm{S}$ & $1 / \mathrm{R}$ \\
III combination & $3 / \mathrm{R}$ & $3 / \mathrm{R}$ & $3 / \mathrm{R}$ & $3 / \mathrm{R}$ & $3 / \mathrm{R}$ & $3 / \mathrm{R}$ \\
\hline
\end{tabular}

$\mathrm{AMC}=$ amoxicillin/clavulanate; $\mathrm{CAZ}=$ ceftazidime; $\mathrm{CPD}=$ cefpodoxime; $\mathrm{ATM}=$ aztreonam; $C R O=$ ceftriaxone; $F O X=$ cefoxitin .

Table 3 Correlation of Check-MDR $\mathrm{CT}_{102}$ and phenotypic methods in detection of ESBL isolates

\begin{tabular}{lll}
\hline Methods for detection of ESBLs & $95 \% \mathrm{Cl}$ & $\mathrm{p}$ \\
\hline Check-MDR CT $_{102} /$ double disk synergy test & 41.92 to 54.55 & $\mathrm{p}<0.0001$ \\
Check-MDR CT $_{102} /$ VITEK 2 compact /AES & -4.43 to 5.36 & $\mathrm{p}=1.0$ \\
VITEK 2 compact /AES/double disk synergy test & 40.13 to 52.73 & $\mathrm{p}<0.0001$ \\
\hline
\end{tabular}

Out of 50 isolates which tested positive for genotype ESBL by the molecular test, 48 were positive by the VITEK 2/AES. By applying the Chi ، square test (McNemar) we found that the results of these two tests in detection of ESBL are not significantly different $(95 \% \mathrm{CI}=-4.43$ to $5.36 ; \mathrm{p}=1.0)$. The sensitivity of the VITEK 2/AES was $96 \%$.
Out of 48 isolates which tested positive by the VITEK 2/AES, 21 were also positive by double-disk synergy test. By applying the Chi square test we found that these two tests are significantly different in detection of ESBL (95\% CI=40.13 to 52.73; $\mathrm{p}<0.0001$ ). The susceptibility of the test is $43 \%$ (Table 3 ). 
Table 4 Correlation of molecular tests and resistance of ESBL isolates to amoxicillin/ clavulanate, aztreonam, and cefoxitin

\begin{tabular}{|c|c|c|}
\hline Methods for detection of ESBLs & $95 \% \mathrm{Cl}$ & $\mathrm{p}$ \\
\hline Check-MDR CT $\mathrm{T}_{102} /$ DDST amoxicilline/clavulanate & -6.05 to 9.96 & $p=0.6875$ \\
\hline Check-MDR CT ${ }_{102} / \mathrm{DDST}$ aztreonam & -0.71 to 19.09 & $p=0.065$ \\
\hline Check-MDR CT $_{102} /$ DDST cefoxitin & 23.46 to 41.73 & $\mathrm{p}<0.0001$ \\
\hline
\end{tabular}

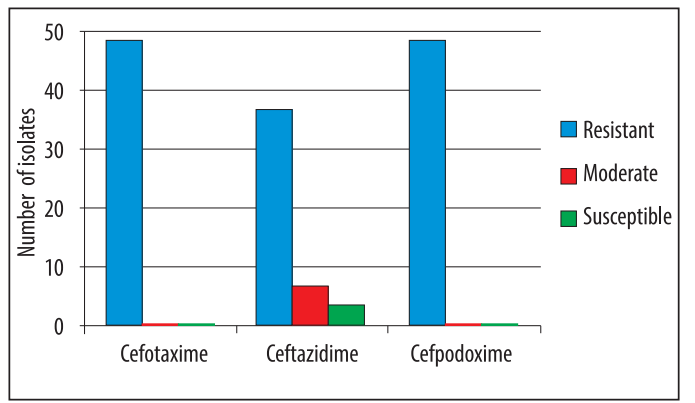

Figure 4 Susceptibility of ESBL isolates confirmed by the molecular test.

\section{Sensitivity of ESBL isolates confirmed by molecular test on the cephalosporin's of III generation}

50 isolates tested positive by molecular test. Out of those, 38 isolates were resistant to ceftazidime, 8 were intermediary susceptible and 4 were susceptible. All 50 isolates was resistant to ceftriaxon and cefpodoxime.

By applying the Chi square test, we found a statistically significant difference (95\% $\mathrm{CI}=6.60$ to $23.54, \mathrm{p}=0.0034$ ) between the results of the molecular test (where a positive result implies resistance to third-generation cephalosporins) and resistance to ceftazidime, whereas no statistical difference was found between the resistance to cefpodoxime and ceftriaxone (50/50) compared to the results of the molecular test (Figure 4).

\section{Resistance of ESBL isolates to amoxicilline/ clavulonate, aztreonam and cefoxitin confirmed by the molecular test}

Considering 50 isolates confirmed by the molecular test, we applied the Chi square test and did not find a statistically signifi- cant difference between the results of the molecular test and resistance to amoksicillin/clavulonate (95\% CI=-6.05 to 9.96; $\mathrm{p}=0.6875)$, and aztreonam ( $95 \% \mathrm{CI}=-0.71$ to 19.09; $\mathrm{p}=0.0654$ ) but we found a statistically significant difference between the results of molecular test and cefoxitin ( $95 \% \mathrm{CI}=23.46$ to 41.73; $\mathrm{p}<0.0001$ ) (Table 4).

\section{Discussion}

The number of newly discovered $B$-lactamases from Enterobacteriaceae has been rising annually, increasing the need for the introduction of new methods for their detection.

Many existing methods for detection of ESBL, AmpC or carbapenemases with Enterobacteriaceae and slow-fermentative Gram negative bacilli are technically demanding and their performance requires a great deal of time and special skills in a microbiologist. Small laboratories, which do not have appropriate equipment for molecular confirmation of individual types of resistance, must have a sensitive and specific method available for phenotypic detection of the same. According to the CLSI recommendations in M100-S21 Vol. 31 No. 1, phenotypic detection of ESBL by disk diffusion or broth dilution is based on the increased activity of cefotaxime or ceftazidime in the presence of clavulanic acid. Commercial methods, such as Etest, VITEK 2 and Phoenix, have developed phenotypic ESBL tests using CLSI methods as the reference comparative method. Molecular detection is based on the detection of resistance genes or 
their products. Results of phenotypic methods for detection of ESBL isolates are relative, and applying different systems gives different results. Genotypic methods can detect resistance but lack of resistance does not indicate susceptibility. Resistance proven in this way is absolute - it is either present or not.

Application of the disk diffusion method with ceftazidime, amoxicillin/clavulanate, and cefotaxime disks, which are placed 30 $\mathrm{mm}$ from the center of one to the center of another disk, was first described by Jarlier et al. in 1988. This method has remained a reliable method in clinical laboratories, although reading is sometimes difficult. The sensitivity of this test can be increased if disks are set at the distance of $30 \mathrm{~mm}$ and 20 $\mathrm{mm}$ (7). In our study we used $20 \mathrm{~mm}$ disk spacing.

The sensitivity of a screening test for the detection of ESBLs with Enterobacteriaceae varies, depending on the antibiotic used as a predictor of their existence. Application of more than one antibiotic increases the sensitivity of the test. Cefpodoxime and ceftazidime show the greatest sensitivity for detection of ESBLs. Phenotypic confirmation tests do not detect all ESBLs. Besides ESBLs, some bacteria possess other $\beta$-lactamases which can mask detection of ESBLs during phenotypic testing, resulting in false-negatives. This includes AmpC $\beta$-lactamases and TEM $\beta$-lactamases resistant to inhibitors. Hyper production of TEM and SHV $\beta$-lactamases in bacteria with ESBLs can also give falsenegative results in phenotypic confirmation tests. This is why it is necessary to apply molecular detection methods to detect bacteria with the presence of multiple $\beta$-lactamases. However, molecular methods are not available in all laboratories (5).

The susceptibility of double-disk synergy test in our research was $48 \% .50$ isolates tested by the molecular test were positive for genotype ESBL. Out of those, 21 were also positive by double-disk synergy test. We found that the results of these two tests in detection of ESBL are significantly different ( $95 \% \mathrm{CI}=41.92$ to $54.55 ; \mathrm{p}<0.0001)$. In our study we found that DDST failed in AMC R isolates and we assume that narrowing the disk spacing might improve ESBL detection in AMC R isolates. The reason for these results is probably due to the joined resistance we found using VITEK 2/AES.

According to the recommendations of Livermore and Woodford from 2004, determining the susceptibility of isolated bacteria to ceftazidime and cefotaxime or to cefpodoxime should be the first line in detection of ESBLs. A confirmatory test should be done when resistance to any of the above mentioned antibiotics is found. To confirm the mechanism of resistance, it is very important to identify the organism to the species level, or at least for those isolates that are resistant to the above mentioned antibiotics. This is necessary because with Klebsiella pneumoniae and Escherichia coli, the cefpodoxime clavulanate combined disk is used as a confirmation test for the detection of ESBL, while with Enterobacter species and Citrobacter freundii, the cefpirome/clavulanate combination disk is used. According to the same author, the best choice of a cephalosporin is the one that can reveal all ESBLs, even when their production is rare, because since 2001, CTX-M enzymes, as well as TEM and SHV mutants, have been isolated in clinical and in outpatient specimens. For this reason, the best choice for detection of TEM and SHV ESBLs is resistance to ceftazidime and variable to cefotaxime, CTX-M ESBLs, are always resistant to cefotaxime and variable to ceftazidime and for all ESBLs resistance to cefpodoxime is compulsory (8). In our research, comparing disk diffusion and Check-MDR CT ${ }_{102}$, we defined that the best predictor for ESBLs is resistance to cefpodoxime and ceftriaxone, because in all 50 molecular test confirmed isolates we found resistance by the double disk synergy 
test, but also we found a statistically significant difference between the results of the molecular test and resistance to cefoxitin using disk diffusing testing ( $95 \% \mathrm{CI}=23.46$ to 41.73 ; $\mathrm{p}<0.0001)$. Out of $50 \mathrm{ESBL}$ isolates confirmed by molecular tests, 28 isolates showed sensitivity to cefoxitin, 14 isolates had intermediate sensitivity and 8 isolates showed resistance. It was not a good predictor of ESBLs

Resistance to ceftazidime is used in practice to indicate the presence of an ESBL, and ceftazidime is the best substrate for TEM and SHV ESBLs. When ceftazidime is used alone in practice, then CTX-M producing isolates will not be detected, since they are susceptible to ceftazidime. Many ESBLs show an "inoculum effect" where MICs of broad-spectrum cephalosporins increase if the inoculum increases. To avoid the possibility of omission in the detection of the CTX-M enzymes, it is important to examine susceptibility to cefotaxime, besides the susceptibility to ceftazidime. Applying the double synergy test in our work, we found a typical phenotypic profile for ESBLs in $42 \%$ (21/50) of ESBL isolates which were confirmed by Check-MDR $\mathrm{CT}_{102}$. However, in $24 \%(12 / 50)$ of ESBL isolates we found that ceftazidime results were intermediate $(8 / 50)$ or susceptible $(4 / 50)$. Such findings can be explained by the fact that the ESBL isolates from our research belonged to the CTX-M genotype, for which ceftazidime zone diameters are intermediate or susceptible in in vitro conditions (9).

In the study of Peer et al., (10), $10.8 \%$, 9.5\% and $5.4 \%$ of ESBL producing bacteria had false susceptibility to third-generation cephalosporins (ceftazidime, cefotaxime and ceftriaxone) with interpretation of a routine disk diffusion method. In our research, $4 / 50$ isolates ( $8 \%)$, confirmed by the molecular method, had false susceptibility to ceftazidime and none of the isolates had false susceptibility to ceftriaxone using DDST and VITEK 2/AES.
According to Peer et al. (10), usage of more than one antibiotic, such as ceftazidime, cefotaxime, ceftriaxone, aztreonam and cefpodoxime, significantly improves the sensitivity of ESBL detection. The same authors underline that using DDST, (double-disk synergy test), PCDDTs (Phenotypic Confirmatory Disk Diffusion Tests) and cefoxitin for the screening of ESBL/AmpC beta-lactamases in positive Klebsiella isolates, resulted in ESBL detection for 69.5\% (64/92) of the isolates, AmpC beta-lactamase detection in $19.5 \%$ (18/92) of the isolates, and detection of both mechanisms of resistance in $10.8 \%(10 / 92)$ of the isolates (10).

Although specificity of DDST is well documented, its sensitivity is variable and varies between $76.5 \%, 93.3 \%, 87 \%$ and $79 \%$ in different studies. This difference in DDST sensitivity appears in different studies because standards set for performance of DDST are often complicated and very precise. Different DDST sensitivity also appears because of the application of different disks (11).

In our research, out of 55 isolates examined with VITEK 2/AES (ESBL screening test includes the resistance on one of III generation cephalospofins) we found ESBL confirmation in $89.09 \%(49 / 55)$ of the isolates and in $10.9 \%(6 / 55)$ we found two phenotypes, AmpC and ESBL. However, after application of the molecular method CheckMDR CT $_{102}$ for ESBL detection, we obtained a positive result in $90.90 \%(50 / 55)$ isolates. With these isolates and using the double disk synergy test, we detected an ESBL phenotype in $42 \%(21 / 50)$ of the isolates. VITEK 2 /AES detected an ESBL/AmpC phenotype in 6 isolates and there was a phenotypic profile corresponding to the presence of $\mathrm{AmpC}$ $\beta$ lactamases in $3 / 6$ (50\%) of the isolates by double disk synergy test.

In a multicenter evaluation of the VITEK 2 Advanced Expert System for interpretive reading of antimicrobial resistance, Livermore et al. determined that for 126 of 
137 reference genotypes there was a phenotypic match with the results obtained using the VITEK 2 Advanced Expert System; in 4 isolates there was a partial match, while in 6 isolates the results were discrepant. With 1 isolate the result could not be interpreted. The same study proved that discrepancies were found in only 64 of 963 interpretations in 10 European countries, which indicates that errors are minimal if the VITEK 2 and AES systems are used in an appropriate manner (12). In our research, out of 50 isolates which tested positive for genotype ESBL by the molecular test, 48 were positive by the VITEK 2/AES. By applying the Chi square test, we found that the results of these two tests in detection of ESBL are not significantly different $(\mathrm{p}=1.0)$. The sensitivity of the VITEK 2/AES is $96 \%$.

To analyze the sensitivity of the VITEK 2 ESBL test, in Spanu et al. (2006), as well as our research, molecular ESBL identification was applied as a confirmation test and the sensitivity of the VITEK 2 ESBL test was found to be $98.1 \%$ (306/312). False positive results were found in $0.24 \%(2 / 817)$. In this research, out of the 50 positive results obtained by Check-MDR CT102, 48 were positive on the VITEK 2 Compact system and sensitivity was $96 \%$. In 2 isolates which were positive by Check-MDR CT102, VITEK 2/ AES detected the AmpC/ESBL phenotype. In 5/55 isolates that were negative by CheckMDR CT102, the AmpC/ESBL phenotype (VITEK 2/AES) was found in 4/5 (80\%). The ESBL phenotype was detected by VITEK $2 /$ AES in $1 / 5(20 \%)$ of Check-MDR CT102 negative isolates. From our results and from results obtained by other authors, it can be concluded that VITEK 2 is a fast and reliable/practical tool for the routine identification of ESBL producing Enterobacteriaceae isolates (13).

\section{Limitations of study}

During preparation of this work we had some limitations. One of them was the study's small sample size, which is a consequence of the inability to purchase a set of performance analysis of a large number of molecular tests, and the other was the domination of one ESBL type.

\section{Conclusion}

In conclusion, this study shows that in addition to molecular methods, which reliably detect genes for each individual mechanism of resistance in routine daily testing, good detection of ESBL-producing bacteria, especially CTX-M, can be obtained with phenotypic methods i.e. microdilution method in VITEK 2 Compact apparatus with AES and the disk diffusion test with a cefpodoxime, and ceftriaksone disk.

Authors' contributions: Conception and design: FN, $\mathrm{MH}$; Acquisition, analysis and interpretation of data: FN, MG; Drafting the article: FN, ZD, SP; Revising it critically for important intellectual content: $\mathrm{FN}, \mathrm{MH}$, NT.

Conflict of interest: The authors declare that they have no conflict of interest.

\section{References}

1. Paterson DL, Bonomo RA. Extended-spectrum beta-lactamases: a clinical update. Clin Microbiol Rev. 2005;18(4):657-86.

2. Philippon A, Labia R, Jacoby G. Extended-spectrum beta-lactamases. Antimicrob Agents Chemother. 1989;33(8):1131-6.

3. Ambler RP, Coulson AF, Frère JM, Ghuysen JM, Joris B, Forsman M, et al. A standard numbering scheme for the class A beta-lactamases. Biochem J. 1991;15;276(Pt 1):269-70.

4. Bush K, Jacoby GA, Medeiros AA. A functional classification scheme for beta-lactamases and its 
correlation with molecular structure. Antimicrob Agents Chemother. 1995;39(6):1211-33.

5. Clinical and Laboratory Standards Institute. Performance Standards for Antimicrobial Susceptibility Testing; Twenty-First Informational Supplement. CLSI Document M100S21. Wayne (Pennsylvania): Clinical and Laboratory Standards Institute; 2011.

6. European Committee on Antimicrobial Susceptibility Testing (EUCAST). Breakpoint tables for interpretation of MICs and zone diameters. Version 1.3. EUCAST; 5 Jan 2011. [updated 2012 December 1]. Available from: http://www.eucast. org/fileadmin/src/media/PDFs/EUCAST_files/ Disk_test_documents/EUCAST_breakpoints_ v1.3_pdf.pdf.

7. Thomson KS, Sanders CC. Detection of extendedspectrum beta-lactamases in members of the family Enterobacteriaceae: comparison of the doubledisk and three-dimensional tests. Antimicrob Agents Chemother. 1992;36(9):1877-82.

8. Livermore DM, Woodford N. Laboratory detection and reporting of bacteria with extended spectrum ßlactamases. Colindale, London: Antibiotic Resistance Monitoring and Reference Laboratory, Specialist and Reference Microbiology Division, Health Protection Agency; 2004. [updated 2012 October 16]. Available from: http:// www.hpa.org.uk/ webc/HPAwebFile/HPAweb_C/1194947314416.
9. Brenwald NP, Jevons G, Andrews JM, Xiong JH, Hawkey PM, Wise R. An outbreak of a CTX-Mtype beta-lactamase-producing Klebsiella pneumoniae: the importance of using cefpodoxime to detect extended-spectrum beta-lactamases. J Antimicrob Chemother. 2003;51(1):195-6.

10. Peer MA, Manzoor AT, Fomda BA, Kaiser A. Extended Spectrum- $\beta$-Lactamase producing Klebsiella pneumoniae at a tertiary care setup in Kashmir, India: Comparative phenotypic detection and antimicrobial susceptibility pattern. RIF. 2010;1(2):124-33.

11. Duttaroy B, Mehta S. Extended spectrum b lactamases (ESBL) in clinical isolates of Klebsiella pneumoniae and Escherichia coli. Indian J Pathol Microbiol. 2005;48(1):45-8.

12. Livermore DM, Struelens M, Amorim J, Baquero F, Bille J, Canton R, et al. Multicentre evaluation of the VITEK 2 Advanced Expert System for interpretive reading of antimicrobial resistance tests. J Antimicrob Chemother. 2002;49(2):289-300.

13. Spanu T, Sanguinetti M, Tumbarello M, D’Inzeo T, Fiori B, Posteraro B, et al. Evaluation of the new VITEK 2 extended-spectrum beta-lactamase (ESBL) test for rapid detection of ESBL production in Enterobacteriaceae isolates. J Clin Microbiol. 2006;44(9):3257-62. 